\title{
A Uniform Syntactical Method for Proving Coinduction Principles in $\lambda$-calculi ${ }^{\star}$
}

\author{
Marina Lenisa \\ Dipartimento di Matematica e Informatica, Università di Udine, Italy. \\ lenisa@dimi.uniud.it
}

\begin{abstract}
Coinductive characterizations of various observational congruences which arise in the semantics of $\lambda$-calculus, when $\lambda$-terms are evaluated according to various reduction strategies, are discussed. We analyze and extend to non-lazy strategies, both deterministic and nondeterministic, Howe's congruence candidate method for proving the coincidence of the applicative (bisimulation) and the contextual equivalences. This purely syntactical method is based itself on a coinductive argument.
\end{abstract}

\section{Introduction}

This paper is part of a general project aiming at finding elementary proof $p r_{i n-}$ ciples for reasoning rigorously on infinite computational objects, see [4, 9] for the case of higher order functions, and [8] for the case of higher order processes. In this paper, as in $[4,9]$, we focus on the behaviour of $\lambda$-terms when these qre evaluated according to various reduction strategies. We address the problem of showing the coincidence of the applicative (bisimulation) equivalence with the $\mathfrak{b} b$ servational (operational, contextual) equivalence for various reduction strategi es, thus deriving a coinduction principle for establishing obsevational equivalences. In particular, in this paper we analyze and generalize to non-lazy strategies the purely syntactical method originally introduced by Howe $([6,7])$ for lazy fur f $_{\mathrm{l}}$ tional languages. We call this method congruence candidate method.

A reduction strategy is a procedure for determining, for each $\lambda$-term, a specific $\beta$-redex in it, to contract. Let $\Lambda(C)\left(\Lambda^{0}(C)\right)$ denote the set of (closed) $\lambda$-tern ts, $_{1 \mathrm{~s}}$ where $C$ is a set of base constants. When $C=\emptyset$, we write $\Lambda\left(\Lambda^{0}\right)$. A (possibily non-deterministic) strategy can be formalized as a relation $\rightarrow_{\sigma} \subseteq A(C) \times A(\mathcal{Y})$ $\left(\Lambda^{0}(C) \times \Lambda^{0}(C)\right)$ such that, if $(M, N) \in \rightarrow_{\sigma}$ (also written infix as $M \rightarrow_{\sigma} N$ ), then $N$ is a possible result of applying $\rightarrow_{\sigma}$ to $M$. The set of terms which do not belong to the domain of $\rightarrow_{\sigma}$ are partitioned into two disjoint sets: the set of $\sigma$-values, denoted by $V a l_{\sigma}$, and the set of $\sigma$-deadlocks. Given $\rightarrow_{\sigma}$, we can define the evaluation relation $\Downarrow_{\sigma} \subseteq \Lambda(C) \times \Lambda(C)\left(\Lambda^{0}(C) \times \Lambda^{0}(C)\right)$, such that $M \Downarrow_{\sigma} N$ holds if and only if there exists a (possible empty) reduction path from $M$ to a $\sigma$-value $N$. If there exists $N$ such that $M \Downarrow_{\sigma} N$, then $\rightarrow_{\sigma}$ halts successfully on $M$ and $M$ converges $\left(M \Downarrow_{\sigma}\right)$, otherwise $\rightarrow_{\sigma}$ does not terminate on $M$ or reaches a deadlock from $M$, and $M$ diverges $\left(M \psi_{\sigma}\right)$. Each reduction strategy induces án

* Work supported by HCM Contract No. CHRX-CT92.0046 Lambda Calcul Typé, 
operational semantics, in that we can imagine a machine which evaluates terms by implementing the given strategy. The observational equivalence arises if we consider programs as black boxes and only observe their "halting properties".

Definition 1 ( $\sigma$-observational Equivalence). Let $\rightarrow_{\sigma}$ be a reduction strategy and let $M, N \in \Lambda^{0}(C)$. The observational equivalence $\approx_{\sigma}$ is defined by $M \approx_{\sigma} N$ iff $\forall C[] .\left(C[M], C[N] \in \Lambda^{0}(C) \Rightarrow\left(C[M] \Downarrow_{\sigma} \Leftrightarrow C[N] \Downarrow_{\sigma}\right)\right)$.

Showing $\sigma$-equivalences by induction on computation steps is difficult. Powerful proof-principles, allowing to factorize this difficult task, are precious. Coinduction principles for establishing $\approx_{\sigma}$ follow from the fact that $\approx_{\sigma}=\approx_{\sigma}^{a p p}$, where $\approx_{\sigma}^{a p p}$ denotes the applicative equivalence induced by $\rightarrow_{\sigma}$ (see Definition 2). It is interesting to notice that these two equivalences do not coincide for all strategies, see [9] for counterexamples.

The proof of $\approx_{\sigma} \supseteq \approx_{\sigma}^{a p p}$ can be factorized into two steps:

1. $\approx_{\sigma}^{a p p}$ is a congruence w.r.t. application;

2. $\approx_{\sigma}^{a p p}$ is a congruence w.r.t. $\lambda$-abstraction.

In many cases step 2 is not difficult to prove, while step 1 is in general problematic to show, and requires a specific technique. In this paper, we discuss the congruence candidate method for proving step 1 . This method was originally introduced for the lazy call-by-name reduction strategy in [6], and later generalized to a class of lazy strategies by-name and by-value in [7]. Here we extend the method so as to deal with non-lazy strategies, both deterministic and nondeterministic, whose evaluation relation needs to be defined on the whole set of $\lambda$-terms and hence it has to deal also with reduction of open terms. The congruence candidate method is based on the definition of a "candidate relation", which is a congruence w.r.t. application, and which extends $\approx_{\sigma}^{a p p}$. Reasoning by coinduction, one shows that this relation coincides with $\approx_{\sigma}^{a p p}$; hence $\approx_{\sigma}^{a p p}$ is itself a congruence w.r.t. application. This method can be applied successfully to various reduction strategies in the literature, thus providing alternative proofs to those in [9], to the conjectures in [4].

In this paper we use $\lambda$-calculus concepts and notation as defined in $[2,4]$. The paper is organized as follows. In Section 1 we introduce the problem of characterizing coinductively contextual equivalences via applicative equivalences. In Section 2 we present a list of strategies. In Section 3 we present in general the congruence candidate method, and we derive a proof of $\approx_{\sigma}=\approx_{\sigma}^{a p p}$ for all the strategies of Section 2. Final remarks appear in section 4.

The author is grateful to F. Honsell and A. Pitts for useful discussions.

\section{Coinductive Characterizations via Applicative Equivalences}

Given a reduction strategy $\rightarrow_{\sigma}$, the $\sigma$-applicative equivalence, $\approx_{\sigma}^{a p p}$, is defined by testing programs only on applicative (closed) contexts. It is reminiscent of bisimilarity in concurrent languages ([1]). 
Definition 2. Let $\approx_{\sigma}^{a p p} \subseteq \Lambda^{0}(C) \times \Lambda^{0}(C)$ be the applicative equivalence: $M \approx_{\sigma}^{a p p} N \Leftrightarrow \forall P_{1}, \ldots, P_{n} \in \Lambda^{0}(C), n \geq 0 .\left(M P_{1} \ldots P_{n} \Downarrow_{\sigma} \Leftrightarrow N P_{1} \ldots P_{n} \Downarrow_{\sigma}\right)$.

The equivalence $\approx_{\sigma}^{a p p}$ has a coinductive characterization:

Lemma 1. The applicative equivalence $\approx_{\sigma}^{a p p}$ can be viewed as the greatest fixed point of the monotone operator $\Psi_{\sigma}: \mathcal{P}\left(\Lambda^{0}(C) \times \Lambda^{0}(C)\right) \rightarrow \mathcal{P}\left(\Lambda^{0}(C) \times \Lambda^{0}(C)\right)$ $\Psi_{\sigma}(R)=\left\{(M, N) \mid\left(M \Downarrow_{\sigma} \wedge N \Downarrow_{\sigma} \wedge \forall P \in \Lambda^{0}(C) .(M P, N P) \in R\right) \vee\right.$ $\left.\left(M \Downarrow_{\sigma} \wedge N \Downarrow_{\sigma} \wedge \forall P \in \Lambda^{0}(C) .(M P, N P) \in R\right)\right\}$.

An immediate consequence is the validity of the coinduction principle:

$$
\frac{(M, N) \in R \quad R \text { is a } \Psi_{\sigma} \text {-bisimulation }}{M \approx_{\sigma}^{a p p} N}
$$

where a $\Psi_{\sigma}$-bisimulation is a relation $R \subseteq \Lambda^{0}(C) \times \Lambda^{0}(C)$ s.t. $R \subseteq \Psi_{\sigma}(R)$.

If $\approx_{\sigma}=\approx_{\sigma}^{a p p}$, then the coinduction principle above can be used to establish directly the observational equivalence. Hence the natural question arises: for which strategies $\sigma$ 's do the two equivalences coincide? Notice that there are $\sigma$ 's such that $\approx_{\sigma} \nsupseteq \approx_{\sigma}^{a p p}$, see [9] for counterexamples. However, for many interesting strategies in the literature, one can show that $\approx_{\sigma}=\approx_{\sigma}^{a p p}$, see e.g. $[1,3,4,7,12,10]$. In general, proofs of the coincidence of the two equivalences are rather difficult and apply only to specific strategies. The technique discussed in Section 3 is rather general and it can be used for establishing the coincidence for all the strategies of Section 2.

\section{A List of Strategies}

In this section we present a list of reduction strategies, together with the corresponding evaluation relations.

$\rightarrow_{l}$ strategy. The lazy call-by-name strategy $\rightarrow_{l} \subseteq \Lambda^{0} \times \Lambda^{0}$ reduces the leftmost $\beta$-redex not appearing in a $\lambda$-abstraction. $\operatorname{Val}_{l}=\{\lambda x . M \mid M \in \Lambda\} \cap \Lambda^{0}$. The evaluation $\Downarrow_{l}$ is the least binary relation over $\Lambda^{0} \times V a l_{l}$ satisfying the rules:

\section{$\overline{\lambda x . M \Downarrow_{l} \lambda x . M}$

$$
\frac{M \Downarrow_{l} \lambda x . P \quad P[N / x] \Downarrow_{l} Q}{M N \Downarrow_{l} Q}
$$

Classical $\beta$-reduction is correct w.r.t. $\approx_{l}^{2}$ (see [1]).

$\rightarrow_{v}$ strategy. Plotkin's lazy call-by-value strategy $\rightarrow_{v} \subseteq \Lambda^{0} \times \Lambda^{0}$ reduces the leftmost $\beta$-redex, not appearing within a $\lambda$-abstraction, whose argument is a $\lambda$ abstraction. $V a l_{v}=\{\lambda x . M \mid M \in \Lambda\} \cap \Lambda^{0}$. The evaluation $\Downarrow_{v}$ is the least binary relation over $\Lambda^{0} \times V a l_{v}$ satisfying the following rules:

$\overline{\lambda x . M \Downarrow_{v} \lambda x . M}$

$$
\frac{M \Downarrow_{v} \lambda x . P \quad N \Downarrow_{v} Q \quad P[Q / x] \Downarrow_{v} U}{M N \Downarrow_{v} U}
$$
The $\beta$-reduction $\rightarrow_{\beta_{r}}$ is correct w.r.t. $\approx_{\sigma}$ if $M=\beta_{\beta_{r}} N \Longrightarrow M \approx_{\sigma} N$, where $=_{\beta_{r}}$ is
the $\beta_{r}$-conversion. 
The notion of $\beta$-reduction which is correct w.r.t. $\approx_{v}$ is the $\rightarrow_{\beta_{v}} \subseteq A \times \Lambda$, i.e.: $(\lambda x . M) N \rightarrow \beta_{v} M[N / x], \quad$ if $N$ is a variable or an abstraction.

$\rightarrow_{0}$ strategy. Let $\Omega$ be a new constant. The non-deterministic strategy $\rightarrow_{o} \subseteq$ $\Lambda^{0}(\{\Omega\}) \times \Lambda^{0}(\{\Omega\})([5])$ rewrites $\lambda$-terms which contain occurrences of the constant $\Omega$ by reducing any $\beta$-redex. $V a l_{o}=\Lambda^{0}$. Normal forms which are not in $V a l_{o}$ are the $\rightarrow_{o}$-deadlock terms. The evaluation relation $\Downarrow_{o}$ is the least binary relation over $\Lambda^{0}(\{\Omega\}) \times V a l_{o}$ satisfying the following rules:

$\frac{M \in V a l_{o}}{M \Downarrow_{o} M} \quad \frac{C[(\lambda x . M) N] \notin V a l_{o} C[M[N / x]] \Downarrow_{o} P}{C[(\lambda x . M) N] \Downarrow_{o} P}$

$\beta$-reduction is trivially correct w.r.t. $\approx_{o}$.

$\rightarrow_{h}$ strategy. The head call-by-name strategy $\rightarrow_{h} \subseteq \Lambda \times \Lambda$ reduces the leftmost $\beta$-redex, if the term is not in head normal form. $V a l_{h}$ is the set of $\lambda$-terms in head normal form. The evaluation $\Downarrow_{h}$ is the least binary relation over $A \times V a l_{h}$ satisfying the following rules, for $n \geq 0$ :

$\frac{M \Downarrow_{h} N}{x M_{1} \ldots M_{n} \Downarrow_{h} x M_{1} \ldots M_{n}} \quad \frac{M[N / x] M_{1} \ldots M_{n} \Downarrow_{h} P}{\lambda x . M \Downarrow_{h} \lambda x . N} \quad \frac{M M_{1} \ldots M_{n} \Downarrow_{h} P}{(\lambda x . M) N M_{1} \ldots}$

$\beta$-reduction is correct w.r.t. $\approx_{h}$ (see e.g. [2]).

$\rightarrow_{n}$ strategy. The normalizing strategy $\rightarrow_{n} \subseteq \Lambda \times \Lambda$ reduces the leftmost $\beta$ redex. $V a l_{n}$ is the set of $\lambda$-terms in normal form. The evaluation $\Downarrow_{n}$ is the least binary relation over $\Lambda \times V a l_{n}$ satisfying the following rules, for $n \geq 0$ :

$$
\frac{M_{1} \Downarrow_{n} M_{1}^{\prime} \ldots M_{n} \Downarrow_{n} M_{n}^{\prime}}{x M_{1} \ldots M_{n} \Downarrow_{n} x M_{1}^{\prime} \ldots M_{n}^{\prime}} \quad \frac{M \Downarrow_{n} N}{\lambda x \cdot M \Downarrow_{n} \lambda x \cdot N} \quad \frac{M[N / x] M_{1} \ldots M_{n} \Downarrow_{n} P}{(\lambda x . M) N M_{1} \ldots M_{n} \Downarrow_{n} P}
$$

$\beta$-reduction is correct w.r.t. $\approx_{n}$.

$\rightarrow_{p}$ strategy. Barendregt's perpetual strategy $\rightarrow_{p} \subseteq \Lambda \times \Lambda$ reduces the leftmost $\beta$-redex not in the operator of a redex, which is either an $I \beta$-redex, or a $K \beta$ redex whose argument is a normal form. $V a l_{p}$ is the set of $\lambda$-terms in normal form. One can easily show that the evaluation $\Downarrow_{p}$ is the least binary relation over $\Lambda \times \mathrm{Val}_{p}$ satisfying the following rules, for $n \geq 0$ :

$$
\frac{M_{1} \Downarrow_{p} M_{1}^{\prime} \ldots M_{n} \Downarrow_{p} M_{n}^{\prime}}{x M_{1} \ldots M_{n} \Downarrow_{p} x M_{1}^{\prime} \ldots M_{n}^{\prime}} \quad \frac{M \Downarrow_{p} N}{\lambda x . M \Downarrow_{p} \lambda x . N} \quad \frac{N \Downarrow_{p} M[N / x] M_{1} \ldots M_{n} \Downarrow_{p} V}{(\lambda x . M) N M_{1} \ldots M_{n} \Downarrow_{p} V}
$$

The reduction $\rightarrow \beta_{K N}$, defined as follows, is correct w.r.t. $\approx_{p}$ :

$(\lambda x . M) N \rightarrow \beta_{K N} M[N / x], \quad$ if $(\lambda x . M) N$ is either an $I \beta$-redex or a $K \beta$-redex whose argument is a closed normal form.

\subsection{General Formats}

The above strategies can be grouped under three general formats: 
Lazy Strategies. $\rightarrow_{l}, \rightarrow_{v}$ can be viewed as special cases of the general format of lazy strategy on a $\lambda$-calculus with variables by name and by values (see $[6,7])$.

Eager Leftmost Strategies. $\rightarrow_{h}, \rightarrow_{n}$, and $\rightarrow_{p}$ are eager in the sense that they reduce under the scope of a $\lambda$-abstraction. They can be viewed as special instances of the following general format:

$$
\begin{aligned}
& \frac{M_{i_{1}} \Downarrow_{\sigma} M_{i_{1}}^{\prime} \ldots M_{i_{n}} \Downarrow_{\sigma} M_{i_{n}}^{\prime}}{x M_{1} \ldots M_{k} \Downarrow_{\sigma} x M_{1}^{\prime} \ldots M_{k}^{\prime}} i_{1}, \ldots, i_{n} \in\{1, \ldots, k\}, n \geq 0 \quad \frac{M \Downarrow_{\sigma} N}{\lambda x . M \Downarrow_{\sigma} \lambda x . N} \\
& \frac{M[N / x] M_{1} \ldots M_{n} \Downarrow_{\sigma} V \quad\left(N \Downarrow_{\sigma}\right)}{(\lambda x . M) N M_{1} \ldots M_{n} \Downarrow_{\sigma} V} n \geq 0, \quad \text { where }\left(N \Downarrow_{\sigma}\right) \text { can be omitted. }
\end{aligned}
$$

Non-deterministic Strategies. $\rightarrow_{o}$ can be viewed as a special case of the following general format: let $\emptyset \subset V a l \subset \Lambda(\{C\})$ be closed under $\beta$-reduction,

$$
\frac{M \in V a l}{M \Downarrow_{\sigma} M} \quad \frac{C[(\lambda x . M) N] \notin V a l \quad C[M[N / x]] \Downarrow_{\sigma} P}{C[(\lambda x . M) N] \Downarrow_{\sigma} P}
$$

Notice that there are many ways to extend $\rightarrow_{0}$ on open terms in order to get a strategy of the above format; we will take the natural one.

\section{Showing $\approx_{\sigma}^{a p p}=\approx_{\sigma}$}

In this section, we present in detail the congruence candidate method for establishing $\approx_{\sigma}^{a p p}=\approx_{\sigma}$. A special instance of this method was first used by Howe in the case of the lazy call-by-name strategy $\rightarrow_{l}([6])$, and later generalized to a class of lazy strategies by-name and by-value, including $\rightarrow_{v}$ ([7]). Here we extend Howe's original method so as to deal with more complex strategies, like the eager leftmost strategies, whose evaluation relations cannot be axiomatized only on closed $\lambda$-terms, and non-deterministic strategies, such as $\rightarrow_{o}$. The congruence candidate method is used to show that $\approx_{\sigma}^{a p p}$ is a congruence w.r.t. application. In fact, in order to prove that $\approx_{\sigma}^{a p p} \subseteq \approx_{\sigma}$, it is sufficient to show (Theorem 4): 1. $\approx_{\sigma}^{a p p}$ is a congruence w.r.t. application, i.e. for all $M, N, P, Q \in \Lambda^{0}(C)$,

$$
M \approx_{\sigma}^{a p p} N \wedge P \approx_{\sigma}^{a p p} Q \Longrightarrow M P \approx_{\sigma}^{a p p} N Q
$$

2. $\approx_{\sigma}^{a p p}$ is a congruence w.r.t. $\lambda$-abstraction, i.e., $\forall M, N \in \Lambda(C)$ such that $F V(M, N) \subseteq\left\{x_{1}, \ldots, x_{n}\right\}, \forall P_{1}, \ldots, P_{n} \in \Lambda^{0}(C) . M\left[P_{i} / x_{i}\right] \approx_{\sigma}^{a p p} N\left[P_{i} / x_{i}\right] \Longrightarrow$ $\lambda x_{1} \ldots x_{n} . M \approx_{\sigma}^{a p p} \lambda x_{1} \ldots x_{n} . N$. (In case the strategy is by-value, i.e. for $\sigma=v, p, P_{1}, \ldots, P_{n}$ are chosen to be convergent terms.)

The congruence of $\approx_{\sigma}^{a p p}$ w.r.t. $\lambda$-abstraction is immediate to show, once one has proved the Extensionality of $\approx_{\sigma}^{a p p}$ (see Theorem 2). This is really problematic only for $\sigma=n$; in this case one needs to exploit extensively the separability technique. For lack of space, we omit this proof.

Theorem 2 (Extensionality of $\approx_{\sigma}^{a p p}$ ). i) Let $\sigma=l, v$. Let $M, N \in \Lambda^{0}$ be such that $M \Downarrow_{\sigma}, N \Downarrow_{\sigma}$. If, for all $P \in \Lambda^{0}, M P \approx_{\sigma}^{a p p} N P$, then $M \approx_{\sigma}^{a p p} N$. 
ii) Let $\sigma=o, h, p, n$. Let $M, N \in \Lambda^{0}(C)$. If, for all $P \in \Lambda^{0}(C), M P \approx_{\sigma}^{a p p} N P$, then $M \approx_{\sigma}^{a p p} N$.

Using Theorem 2, one can easily show the following theorem:

Theorem 3. $\approx_{\sigma}^{a p p}$ is a congruence w.r.t. $\lambda$-abstraction, for $\sigma \in\{l, v, o, h, n, p\}$.

Proof. We show that, for $M, N \in \Lambda$ s.t. $F V(M, N) \subseteq\{x\}$,

$\forall P \in \Lambda^{0}$ (possibly convergent). $M[P / x] \approx_{\sigma}^{a p p} N[P / x] \Rightarrow \lambda x . M \approx_{\sigma}^{a p p} \lambda x . N$.

For $\sigma=l, v$ this is immediate. For $\sigma=o, h, n$ the proof follows from the Extensionality Theorem, using the fact, that $(\lambda x . M) P \approx_{\sigma}^{a p p} M[P / x]$, which in turn follows from the correctness of the $\beta$-reduction w.r.t. $\approx_{\sigma}^{a p p}$. For $\sigma=p$, the proof follows from the fact that, for all $M \in \Lambda,\left(\exists P \in \Lambda^{0} . M[P / x] \Downarrow_{p}\right) \Longleftrightarrow M \Downarrow_{p}$.

The implication $(\Rightarrow)$ in this latter fact follows since $\rightarrow_{p}$ is perpetual. The other implication is proved by computation induction, choosing as $P$ a suitable permutator.

Theorem 4. Suppose that $\approx_{\sigma}^{a p p}$ is a congruence w.r.t. $\lambda$-abstraction and application. Then $\approx_{\sigma}^{a p p} \subseteq \approx_{\sigma}$.

Proof. We prove by induction on the context $C[]$ that:

$M \approx_{\sigma}^{a p p} N \Longrightarrow \forall C[]\left(C[M], C[N] \in \Lambda(C) \wedge F V(C[M], C[N]) \subseteq\left\{x_{1}, \ldots, x_{n}\right\} \Rightarrow\right.$ $\left.\forall P_{1} \ldots P_{n} \in \Lambda^{0}(C) . C[M]\left[P_{i} / x_{i}\right] \approx_{\sigma}^{a p p} C[N]\left[P_{i} / x_{i}\right]\right)$.

(In case the strategy is by-value, i.e. $\sigma=v, p, P_{1}, \ldots, P_{n}$ must be convergent terms.)

\subsection{The Congruence Candidate Method}

The aim of the congruence candidate method is to show that $\approx_{\sigma}^{a p p}$ is a congruence w.r.t. application. The main difference between dealing with lazy strategies (whose evaluation relation is axiomatized only on closed $\lambda$-terms) and dealing with eager strategies, like $\rightarrow_{h}, \rightarrow_{n}, \rightarrow_{p}$, lies in the fact that, for eager strategies, in order to show that $\approx_{\sigma}^{a p p}$ is a congruence w.r.t. application, we need to assume that $\approx_{\sigma}^{a p p}$ is a congruence w.r.t. $\lambda$-abstraction. This hypothesis is not needed for the lazy strategies considered in [7]. A further special generalization of the proof is required for non deterministic strategies, like $\rightarrow_{0}$. In fact, the proof of the main proposition in Howe's method proceeds by induction on the length of the derivation of a suitable evaluation judgement, just as we do in the proof of the main proposition for the deterministic strategies in this paper (Propositions 8 and 9). The same result for non deterministic strategies, on the other hand, has to be obtained by induction on the minimal length of a converging path (Proposition 12).

The congruence candidate method is a syntactical method which nonetheless is quite uniform and modular. It makes essential use of the coinduction principle (1) of Section 1, and it is based on the definition of a candidate relation, which is a congruence w.r.t. application, and which extends $\approx_{\sigma}^{a p p}$. The aim is to show that the candidate relation is a $\Psi_{\sigma}$-bisimulation; hence the coinduction principle (1) guarantees that $\approx_{\sigma}^{a p p}$ itself is a congruence w.r.t. application. For the reader's convenience, we outline the: 
General pattern of the congruence candidate method:

- Build a candidate relation ${\widehat{\approx}_{\sigma}^{a p p}}^{a} \subseteq \Lambda(C) \times \Lambda(C)$ s.t.

1. $\widehat{\approx}_{\sigma}^{a p p} \supseteq \approx_{\sigma}^{a p p}$

2 . $\widehat{\approx}_{\sigma}^{a p p}$ is a congruence w.r.t. application;

3. $\left(\widehat{\approx}_{\sigma}^{a p p}\right) \mid \Lambda^{0}(C) \times \Lambda^{0}(C)$ is a $\Psi_{\sigma}$-bisimulation.

- Use the coinduction principle $(1)$ to deduce that $\approx_{\sigma}^{a p p}$ is a congruence w.r.t. application.

More in detail, the congruence candidate method proceeds as follows. First of all, we have to explain how to build the candidate relation $\widehat{\approx}_{\sigma}^{a p p}$. Candidate relations are defined in terms of the extensions to open terms of $\Psi_{\sigma}$-bisimulations:

Definition 3. Let $\eta \subseteq \Lambda^{0}(C) \times \Lambda^{0}(C)$ be a $\Psi_{\sigma}$-bisimulation. Define $\eta^{a} \subseteq \Lambda(C) \times$ $\Lambda(C)$ as follows: let $M, N \in \Lambda(C)$ be s.t. $F V(M, N) \subseteq\left\{x_{1}, \ldots, x_{n}\right\}$,

$M \eta^{a} N \Longleftrightarrow \forall P_{1} \ldots P_{n} \in \Lambda^{0}(C) . M\left[P_{i} / x_{i}\right] \eta N\left[P_{i} / x_{i}\right]$

(In case the strategy is by-value, i.e. for $\sigma=v, p, P_{1}, \ldots, P_{n}$ are chosen to be convergent terms.)

Definition 4 (Candidate Relation). Let $\eta \subseteq \Lambda^{0}(C) \times \Lambda^{0}(C)$ be a reflexive and transitive $\Psi_{\sigma}$-bisimulation. Define the candidate relation $\widehat{\eta} \subseteq \Lambda(C) \times \Lambda(C)$ by induction on $M$ as follows:

$\frac{x \eta^{a} N}{x \hat{\eta} N} \quad \frac{M_{1} \hat{\eta} M_{1}^{\prime} M_{2} \widehat{\eta} M_{2}^{\prime} M_{1}^{\prime} M_{2}^{\prime} \eta^{a} N}{M_{1} M_{2} \widehat{\eta} N} \quad \frac{M \widehat{\eta} M^{\prime} \lambda x \cdot M^{\prime} \eta^{a} N}{\lambda x \cdot M \hat{\eta} N}$

Notice that the candidate relation is not simply the contextual closure of $\eta$; this subtle definition of $\widehat{\eta}$ is necessary to guarantee the crucial Substitutivity Lemma 6. The following lemma is an easy consequence of the definition of $\widehat{\eta}$.

Lemma 5. Let $\eta \subseteq \Lambda^{0}(C) \times \Lambda^{0}(C)$ be a reflexive and transitive $\Psi_{\sigma}$-bisimulation. Then: i) $\hat{\eta}$ is reflexive. ii) $\eta^{a} \subseteq \widehat{\eta}$. iii) $\widehat{\eta}$ is a congruence w.r.t. application. iv) $M \widehat{\eta} M^{\prime} \wedge M^{\prime} \eta^{a} N \Longrightarrow M \widehat{\eta} N$.

Lemma 6 (Substitutivity). $M \widehat{\eta} M^{\prime} \wedge N \widehat{\eta} N^{\prime} \Longrightarrow M[N / x] \widehat{\eta} M^{\prime}\left[N^{\prime} / x\right]$.

(In case the strategy is by-value, i.e. for $\sigma=v, p, N, N^{\prime}$ must be convergent terms.)

Proof. By induction on the structure of $M$.

- $M \equiv x: \quad \frac{x \eta^{a} M^{\prime}}{x \widehat{\eta} M^{\prime}}$

$x \eta^{a} M^{\prime} \Longrightarrow N^{\prime} \eta^{a} M^{\prime}\left[N^{\prime} / x\right]$, from the definition of $\eta^{a}$.

$N \widehat{\eta} N^{\prime} \wedge N^{\prime} \eta^{a} M^{\prime}\left[N^{\prime} / x\right] \Longrightarrow N \widehat{\eta} M^{\prime}\left[N^{\prime} / x\right]$, from item iv of Lemma 5 .

- $M \equiv M_{1} M_{2}: \quad \exists M_{1}^{\prime}, M_{2}^{\prime}$ s.t. $\quad \frac{M_{1} \widehat{\eta} M_{1}^{\prime} M_{2} \widehat{\eta} M_{2}^{\prime} M_{1}^{\prime} M_{2}^{\prime} \eta^{a} M^{\prime}}{M_{1} M_{2} \hat{\eta} M^{\prime}}$

By induction hypothesis, $M_{1}[N / x] \widehat{\eta} M_{1}^{\prime}\left[N^{\prime} / x\right]$ and $M_{2}[N / x] \widehat{\eta} M_{2}^{\prime}\left[N^{\prime} / x\right]$. Moreover, by definition of $\eta^{a}, M_{1}^{\prime} M_{2}^{\prime}\left[N^{\prime} / x\right] \eta^{a} M^{\prime}\left[N^{\prime} / x\right]$. Hence: 
$\frac{M_{1}[N / x] \widehat{\eta} M_{1}^{\prime}\left[N^{\prime} / x\right] \quad M_{2}[N / x] \widehat{\eta} M_{2}^{\prime}\left[N^{\prime} / x\right] \quad M_{1}^{\prime} M_{2}^{\prime}\left[N^{\prime} / x\right] \eta^{a} M^{\prime}\left[N^{\prime} / x\right]}{M_{1} M_{2}[N / x] \widehat{\eta} M^{\prime}\left[N^{\prime} / x\right]}$

- $M \equiv \lambda y \cdot M_{1}: \quad \exists M_{1}^{\prime}$ s.t. $\quad \frac{M_{1} \hat{\eta} M_{1}^{\prime} \lambda y \cdot M_{1}^{\prime} \eta^{a} M^{\prime}}{\lambda y \cdot M_{1} \hat{\eta} M^{\prime}}$

By induction hypothesis, $M_{1}[N / x] \widehat{\eta} M_{1}^{\prime}\left[N^{\prime} / x\right]$. By definition of $\eta^{a}$, $\left(\lambda y . M_{1}^{\prime}\right)\left[N^{\prime} / x\right] \eta^{a} M^{\prime}\left[N^{\prime} / x\right]$. Hence:

$\frac{M_{1}[N / x] \widehat{\eta} M_{1}^{\prime}\left[N^{\prime} / x\right] \quad\left(\lambda y \cdot M_{1}^{\prime}\right)\left[N^{\prime} / x\right] \eta^{a} M^{\prime}\left[N^{\prime} / x\right]}{\left(\lambda y \cdot M_{1}\right)[N / x] \widehat{\eta} M^{\prime}\left[N^{\prime} / x\right]}$.

Thus, if we take $\eta$ to be the equivalence $\approx_{\sigma}^{a p p}$, we get a relation $\approx_{\sigma}^{a p p}$, which, by item ii of Lemma 5 , extends $\approx_{\sigma}^{a p p}$. Moreover, by item iii of the same lemma, it is a congruence w.r.t. application. In order to show that $\approx_{\sigma}^{a p p}$ is itself a congruence w.r.t. application, we prove that $\left(\approx_{\sigma}^{a p p}\right)_{\mid \Lambda^{0}(C) \times \Lambda^{0}(C)}=\approx_{\sigma}^{a p p}$. This is done using the coinduction principle (1), by proving that $\left(\widehat{\approx}_{\sigma}^{a p p}\right)_{\mid A^{0}(C) \times \Lambda^{0}(C)}$ is a $\Psi_{\sigma^{-}}$ bisimulation. Notice that this is the only part of the proof that depends on the reduction strategy $\rightarrow_{\sigma}$. We succeed in showing that $\left(\widehat{\approx}_{\sigma}^{a p p}\right)_{\mid \Lambda^{0}(C) \times \Lambda^{0}(C)}$ is a $\Psi_{\sigma^{-}}$ bisimulation for all the strategies of Section 2. The proof of this fact makes an essential use of the Substitutivity Lemma, and moreover, it requires the validity of some further properties, depending on the strategy $\rightarrow_{\sigma}$. E.g. for eager leftmost strategies we have to assume that $\approx_{\sigma}^{a p p}$ is a congruence w.r.t. $\lambda$-abstraction; for $\rightarrow_{v}$, we need the technical property appearing in Lemma 7 below.

Congruence Candidate Technique for Lazy Strategies For the sake of completeness, we outline briefly the proof of the fact that $\left(\widehat{\approx}_{\sigma}^{a p p}\right)_{\mid \Lambda^{0}(C) \times \Lambda^{0}(C)}$ is a $\Psi_{\sigma}$-bisimulation for $\sigma=l, v$. The strategies $\rightarrow_{l}, \rightarrow_{v}$ are special cases of Howe's general format of lazy strategies, see $[6,7]$ for more details.

Lemma 7. For all $M, N \in \Lambda^{0}$, $\left(M \approx_{v}^{a p p} N \wedge M \Downarrow_{v} V\right) \Longrightarrow \exists U .\left(N \Downarrow_{v} U \wedge V \approx_{v}^{a p p} U\right)$.

Proposition 8. $\left(\approx_{\sigma}^{a p p}\right)_{\mid \Lambda^{0} \times \Lambda^{0}}$ is a $\Psi_{\sigma}$-bisimulation, for $\sigma \in\{l, v\}$.

Proof. (Sketch, see $[6,7]$ for more details.) Let $M\left(\widehat{\approx}_{\sigma}^{a p p}\right)_{\mid \Lambda^{0} \times \Lambda^{0}} N$. From items i and iii of Lemma 5 it follows immediately that, for all $P \in \Lambda^{0}, M P\left(\approx_{\sigma}^{a p p}\right)_{\mid \Lambda^{0} \times \Lambda^{0}} N P$. The difficult part of the proof consists in proving that $M\left(\widetilde{\approx}_{\sigma}^{a p p}\right)_{\left.\right|^{0} \times A^{0}} N \wedge M \Downarrow_{\sigma} \Longrightarrow N \Downarrow_{\sigma}$. This can be shown by induction on the derivation of $M \Downarrow_{\sigma}$, using Lemmata 5, 6, and, for $\sigma=v$, also Lemma 7 .

\section{Congruence Candidate Technique for Eager Leftmost Strategies}

Proposition 9. Let $\rightarrow_{\sigma}$ be a eager leftmost strategy s.t. $\approx_{\sigma}^{a p p}$ is a congruence w.r.t. $\lambda$-abstraction. Then $\left(\widehat{\approx}_{\sigma}^{a p p}\right)_{\left.\right|^{0} \times \Lambda^{0}}$ is a $\Psi_{\sigma}$-bisimulation.

Proof. The only non trivial part of the proof consists in proving that $M\left(\widehat{\approx}_{\sigma}^{a p p}\right)_{\mid \Lambda^{0} \times \Lambda^{0}} N \wedge M \Downarrow_{\sigma} \Longrightarrow N \Downarrow_{\sigma}$.

Since the evaluation relation is axiomatized on the whole $\Lambda$, the above fact cannot be 
proved simply by induction on the derivation of $M \Downarrow_{\sigma}$. However, it follows from the stronger result obtained by dropping the restriction on closed $\lambda$-terms, i.e.:

$M \widehat{\approx}_{\sigma}^{a p p} N \wedge M \Downarrow_{\sigma} \Longrightarrow N \Downarrow_{\sigma}$.

To show this, we proceed by induction on the derivation of $M \Downarrow_{\sigma}$.

- $M \equiv x M_{1} \ldots M_{k}$ : then, by hypothesis $\exists V_{i_{1}}, \ldots, V_{i_{n}}$ s.t.

$\frac{M_{i_{1}} \Downarrow_{\sigma} V_{i_{1}} \ldots M_{i_{n}} \Downarrow_{\sigma} V_{i_{n}}}{x M_{1} \ldots M_{k} \Downarrow_{\sigma} x V_{1} \ldots V_{k}} \quad i_{1}, \ldots, i_{n} \in\{1, \ldots, k\}, n \geq 0$

and $\exists N_{1}, \ldots, N_{k}, N^{0}, \ldots, N^{k-1}$ s.t.

$$
\begin{aligned}
& \frac{\frac{x\left(\approx_{\sigma}^{a p p}\right)^{a} N^{0}}{x{\approx_{\sigma}^{a p p}}^{v} N^{\nu}} M_{1}{\approx_{\sigma}^{a p p}}^{a p} N_{1} N^{0} N_{1}\left(\approx_{\sigma}^{a p p}\right)^{a} N^{1}}{x M_{1} \gtrsim_{\sigma}^{a p p} N^{1}}
\end{aligned}
$$

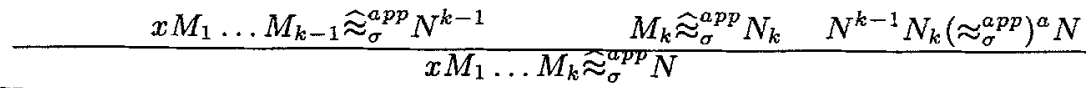

Hence

$x\left(\approx_{\sigma}^{a p p}\right)^{a} N^{0} \Longrightarrow x N_{1}\left(\approx_{\sigma}^{a p p}\right)^{a} N^{0} N_{1}$

$x N_{1}\left(\approx_{\sigma}^{a p p}\right)^{a} N^{0} N_{1} \wedge N^{0} N_{1}\left(\approx_{\sigma}^{a p p}\right)^{a} N^{1} \Longrightarrow x N_{1}\left(\approx_{\sigma}^{a p p}\right)^{a} N^{1}$

$x N_{1} \ldots N_{k}\left(\approx_{\sigma}^{a p p}\right)^{a} N^{k-1} N_{k} \wedge N^{k-1} N_{k}\left(\approx_{\sigma}^{a p p}\right)^{a} N \Longrightarrow x N_{1} \ldots N_{k}\left(\approx_{\sigma}^{a p p}\right)^{a} N$

By induction hypothesis, from $M_{i_{1}} \widehat{\approx}_{\sigma}^{a p p} N_{i_{1}}, \ldots, M_{i_{n}} \widehat{\approx}_{\sigma}^{a p p} N_{i_{n}}$, it follows that $N_{i_{1}} \Downarrow_{\sigma}$ $, \ldots, N_{i_{n}} \Downarrow_{\sigma}$. Thus $x N_{1} \ldots N_{k} \Downarrow_{\sigma}$. Hence, from $x N_{1} \ldots N_{k}\left(\approx_{\sigma}^{a p p}\right)^{a} N$, using the fact that $\approx_{\sigma}^{a p p}$ is a congruence w.r.t. $\lambda$-abstraction, it follows that $N \Downarrow_{\sigma}$.

- $M \equiv \lambda x . M_{1}: \quad$ then, by hypothesis $\exists V_{1}$ s.t. $\frac{M_{1} \Downarrow_{\sigma} V_{1}}{\lambda x \cdot M_{1} \Downarrow_{\sigma} \lambda x \cdot V_{1}}$

and $\exists N_{1}$ s.t. $\quad \frac{M_{1} \widehat{\approx}_{\sigma}^{a p p} N_{1} \lambda x \cdot N_{1}\left(\approx_{\sigma}^{a p p}\right)^{a} N}{\lambda x \cdot M_{1} \widehat{\approx}_{\sigma}^{a p p} N}$.

By induction hypothesis $N_{1} \Downarrow_{\sigma}$. Hence $\lambda x . N_{1} \Downarrow_{\sigma}$. Thus, from $\lambda x . N_{1}\left(\approx_{\sigma}^{a p p}\right)^{a} N$, using the fact that $\approx_{\sigma}^{a p p}$ is a congruence w.r.t. $\lambda$-abstraction, $N \Downarrow_{\sigma}$.

- $M \equiv\left(\lambda x . M_{1}\right) M_{2} \ldots M_{k}$ : then, by hypothesis $\exists V$ s.t.

$\frac{M_{1}\left[M_{2} / x\right] M_{3} \ldots M_{k} \Downarrow_{\sigma} V \quad\left(M_{2} \Downarrow_{\sigma}\right)}{\left(\lambda x \cdot M_{1}\right) M_{2} \ldots M_{k} \Downarrow_{\sigma} V} k \geq 2$

and $\exists N_{1}, \ldots, N_{k}, N^{1}, \ldots, N^{k-1}$ s.t.

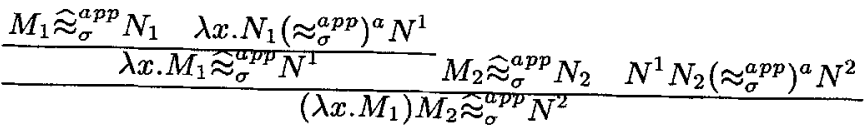

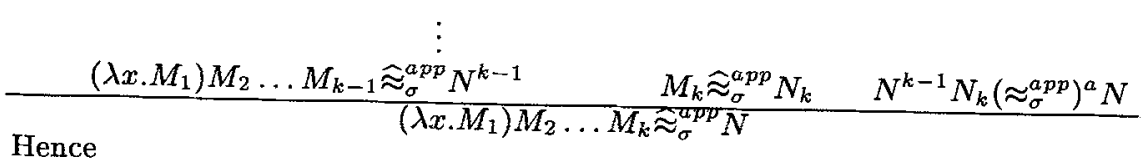




$$
\begin{aligned}
& N^{k-2} N_{k-1}\left(\approx_{\sigma}^{a p p}\right)^{a} N^{k-1} \wedge N^{k-1} N_{k}\left(\approx_{\sigma}^{a p p}\right)^{a} N \underset{N^{k-2}}{\Longrightarrow} N_{k-1} N_{k}\left(\approx_{\sigma}^{a p p}\right)^{a} N \\
& N^{k-3} N_{k-2}\left(\approx_{\sigma}^{a p p}\right)^{a} N^{k-2} \wedge N^{k-2} N_{k-1} N_{k}\left(\approx_{\sigma}^{a p p}\right)^{a} \underset{N^{k-3} N_{k-2} N_{k-1} N_{k}\left(\approx_{\sigma}^{a p p}\right)^{a} N}{ }
\end{aligned}
$$

$N^{1} N_{2}\left(\approx_{\sigma}^{a p p}\right)^{a} N^{2} \wedge N^{2} N_{3} \ldots N_{k}\left(\approx_{\sigma}^{a p p}\right)^{a} N \Rightarrow N^{1} N_{2} \ldots N_{k}\left(\approx_{\sigma}^{a p p}\right)^{a} N$

$\lambda x . N_{1}\left(\approx_{\sigma}^{a p p}\right)^{a} N^{1} \wedge N^{1} N_{2} \ldots N_{k}\left(\approx_{\sigma}^{a p p}\right)^{a} N \Rightarrow\left(\lambda x \cdot N_{1}\right) N_{2} \ldots N_{k}\left(\approx_{\sigma}^{a p p}\right)^{a} N$.

To show that $N \Downarrow_{\sigma}$, it is sufficient to prove that $\left(\lambda x . N_{1}\right) N_{2} \ldots N_{k} \Downarrow_{\sigma}$. Then, from the definition of $\left(\approx_{\sigma}^{a p p}\right)^{a}$, since $\approx_{\sigma}^{a p p}$ is a congruence w.r.t. $\lambda$-abstraction, we get the thesis. To show that $\left(\lambda x . N_{1}\right) N_{2} \ldots N_{k} \Downarrow_{\sigma}$, it is sufficient to prove that $N_{1}\left[N_{2} / x\right] N_{3} \ldots N_{k} \Downarrow_{\sigma}$, and possibly also that $N_{2} \Downarrow_{\sigma}$. This latter fact follows by induction hyp.. To show $N_{1}\left[N_{2} / x\right] N_{3} \ldots N_{k} \Downarrow_{\sigma}$, we proceeds as follows. From $M_{1} \widehat{\approx}_{\sigma}^{a p p} N_{1}, \ldots, M_{k} \widehat{\approx}_{\sigma}^{a p p} N_{k}$, using the Substitutivity Lemma, we get $M_{1}\left[M_{2} / x\right] M_{3} \ldots M_{k} \widehat{\approx}_{\sigma}^{a p p} N_{1}\left[N_{2} / x\right] N_{3} \ldots N_{k}$. Hence, since $M_{1}\left[M_{2} / x\right] M_{3} \ldots M_{k} \Downarrow_{\sigma}$, by induction hypothesis, $N_{1}\left[N_{2} / x\right] N_{3} \ldots N_{k} \Downarrow_{\sigma}$.

\section{Congruence Candidate Technique for Non-deterministic Strategies}

Using the fact that $V a l_{\sigma}$ is closed under $\beta$-reduction, for $\rightarrow_{\sigma}$ non-deterministic strategy of the format of Subsection 2.1, we immediately get

Lemma 10. Let $\rightarrow_{\sigma}$ be a non-deterministic strategy. Then $\beta$-reduction is correct w.r.t. $\approx_{\sigma}$, i.e. for $M, N \in A(C), M=\beta, N \Rightarrow M \approx_{\sigma} N$.

Lemma 11. Let $\rightarrow_{\sigma}$ be a non-deterministic strategy. For all contexts $C[]$, if $C[(\lambda x, P) Q] \widehat{\approx}_{\sigma}^{a p p} N$, then $C\left[P\left[Q / x^{\prime}\right]\right] \widehat{\approx}_{\sigma}^{a p p} N$.

Proof. The proof proceeds by induction on the structure of $C[]$.

- $C[] \in V a r:$ the thesis is immediate.

- $C[] \equiv[]:$ from the hypothesis $(\lambda x . P) Q{\approx_{\sigma}^{\alpha p p}}_{\sigma}, \exists N_{1}, N_{2}, N_{3}$ s.t.

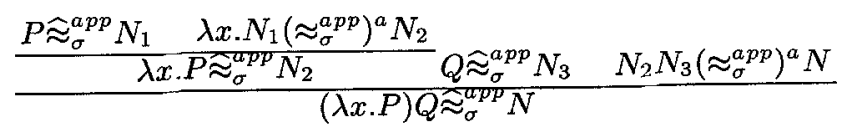

$\lambda x . N_{1}\left(\approx_{\sigma}^{a p p}\right)^{a} N_{2} \wedge N_{2} N_{3}\left(\approx_{\sigma}^{a p p}\right)^{a} N \Longrightarrow\left(\lambda x . N_{1}\right) N_{3}\left(\approx_{\sigma}^{a p p}\right)^{a} N$

using Lemma 10 , we get $N_{1}\left[N_{3} / x\right]\left(\approx_{\sigma}^{a p p}\right)^{a} N$; moreover, by the Substitutivity Lemma, $P \widehat{\approx}_{\sigma}^{a p p} N_{1} \wedge Q \widehat{\approx}_{\sigma}^{a p p} N_{3} \Longrightarrow P[Q / x] \widehat{\approx}_{\sigma}^{a p p} N_{1}\left[N_{3} / x\right]$.

Hence, from $P[Q / x] \widehat{\approx}_{\sigma}^{a p p} N_{1}\left[N_{3} / x\right]$ and $N_{1}\left[N_{3} / x\right]\left(\approx_{\sigma}^{a p p}\right)^{a} N$, using item iv of Lemma 5 , it follows that $P[Q / x] \widehat{\approx}_{\sigma}^{a p p} N$.

- $C[] \equiv C_{1}[] C_{2}[]$ : from the hyp. $C_{1}[(\lambda x . P) Q] C_{2}[(\lambda x . P) Q] \gtrsim_{\sigma}^{a p p} N, \exists N_{1}, N_{2}$ s.t.

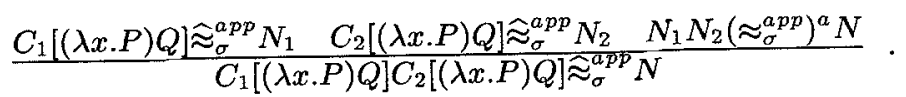

By induction hypothesis, $C_{1}[P[Q / x]] \widehat{\approx}_{\sigma}^{a p p} N_{1}$ and $C_{2}[P[Q / x]] \widehat{\approx}_{\sigma}^{a p p} N_{2}$, hence $C_{1}[P[Q / x]] C_{2}[P[Q / x]] \approx_{\sigma}^{a p p} N_{1} N_{2}$. Then, from $N_{1} N_{2}\left(\approx_{\sigma}^{a p p}\right)^{a} N$, using item iv of Lemma 5 , we get the thesis.

- $C[] \equiv \lambda y . C_{1}[]: \quad$ from the hypothesis $\lambda y . C_{1}[(\lambda x . P) Q] \gtrsim_{\sigma}^{a p p} N, \exists N_{1}$ s.t.

$$
\frac{C_{1}[(\lambda x . P) Q] \widehat{\approx}_{\sigma}^{a p p} N_{1} \quad \lambda y \cdot N_{1}\left(\approx_{\sigma}^{a p p}\right)^{a} N}{\lambda y \cdot C_{1}[(\lambda x . P) Q] \approx_{\sigma}^{a p p} N}
$$


By induction hypothesis, $C_{1}[P[Q / x]] \widehat{\approx}_{\sigma}^{a p p} N_{1}$, hence $\lambda y \cdot C_{1}[P[Q / x]] \widehat{\approx}_{\sigma}^{a p p} \lambda y \cdot N_{1}$. Then, from $\lambda y, N_{1}\left(\approx_{\sigma}^{a p p}\right)^{a} N$, using item iv of Lemma 5 , we get the thesis.

As we remarked earlier, the proof of the fact that $\left(\widehat{\approx}_{\sigma}^{a p p}\right)_{\mid \Lambda^{0} \times \Lambda^{0}}$ is a $\Psi_{\sigma^{-}}$ bisimulation depends essentially on the strategy. The hypotheses of the proposition below have been tuned to the strategy $\rightarrow_{0}$. Different sets of hypotheses are probably necessary to deal with other non-deterministic strategies.

Proposition 12. Let $\rightarrow_{\sigma}$ be a non-deterministic strategy s.t.:

$1 . \approx_{\sigma}^{a p p}$ is a congruence w.r.t. $\lambda$-abstraction;

2. for all $M \in \Lambda(C)$, i) $M \Downarrow_{\sigma} \Longleftrightarrow \lambda x . M \Downarrow_{\sigma}$ and

ii) $\lambda x . M \in V a l_{\sigma} \Longrightarrow M \in V a l_{\sigma}$;

3. for all $M_{1}, M_{2} \in \Lambda(C), \quad$ i) $\left(M_{1} \Downarrow_{\sigma} \wedge M_{2} \Downarrow_{\sigma}\right) \Longrightarrow M_{1} M_{2} \Downarrow_{\sigma}$ and

ii) $M_{1} M_{2} \in V a l_{\sigma} \Longrightarrow\left(M_{1} \in V a l_{\sigma} \wedge M_{2} \in V a l_{\sigma}\right)$, then $\left(\widehat{\approx}_{\sigma}^{a p p}\right)_{\mid \Lambda^{0} \times \Lambda^{0}}$ is a $\Psi_{\sigma}$-bisimulation.

Proof. We prove, by induction on the minimal length $k$ of a convergent path from $M \in \Lambda(C)$, that: $M \approx_{\sigma}^{a p p} N \wedge M \Downarrow_{\sigma} \Longrightarrow N \Downarrow_{\sigma}$.

- Suppose $k=0$. Then we proceed by induction on the structure of $M$ :

$-M \equiv x: \frac{x\left(\approx_{\sigma}^{a p p}\right)^{a} N}{x \widehat{\approx}_{\sigma}^{a p p} N} \quad ;$ from $x\left(\approx_{\sigma}^{a p p}\right)^{a} N$, using hypotheses 1 and $2 \mathrm{i}$ ), we get $N \Downarrow_{\sigma}$.

- $M \equiv \lambda x . M_{1}: \quad \exists N_{1}$ s.t. $\quad \frac{M_{1} \widehat{\approx}_{\sigma}^{a p p} N_{1} \lambda x \cdot N_{1}\left(\approx_{\sigma}^{a p p}\right)^{a} N}{\lambda x \cdot M_{1} \widehat{\approx}_{\sigma}^{a p p} N}$; hence, by hypothesis 2ii), $M_{1} \in V a l_{\sigma}$; from $M_{1} \widehat{\approx}_{\sigma}^{a p p} N_{1}$, using the induction hypothesis, it follows that $N_{1} \Downarrow_{\sigma}$. Hence by hypothesis 2i) $\lambda x . N_{1} \Downarrow_{\sigma}$, and, by hypotheses 1 and $2 \mathrm{i}$ ), $N \Downarrow_{\sigma}$.

- $M \equiv M_{1} M_{2}: \quad \exists N_{1}, N_{2}$ s.t. $\quad \frac{M_{1} \widehat{\approx}_{\sigma}^{a p p} N_{1} M_{2} \widehat{\approx}_{\sigma}^{a p p} N_{2} N_{1} N_{2}\left(\approx_{\sigma}^{a p p}\right)^{a} N}{M_{1} M_{2} \widehat{\approx}_{\sigma}^{a p p} N}$

Since, by hypothesis 3ii), $M_{1}, M_{2} \in V a l_{\sigma}$, by induction hypothesis, $N_{1} \Downarrow_{\sigma}$ and $N_{2} \Downarrow_{\sigma}$, i.e., by hypothesis 3i), $N_{1} N_{2} \Downarrow_{\sigma}$. Hence, by hypotheses 1 and $\left.2 \mathrm{i}\right), N \Downarrow_{\sigma}$.

- Suppose $k>0 . M \equiv C[(\lambda x . P) Q] \rightarrow_{\sigma} C[P[Q / x]] \Downarrow_{\sigma}$ (the length of a minimal convergent path from $C[P[Q / x]]$ is $k-1)$. From $C[(\lambda x . P) Q] \widehat{\approx}_{\sigma}^{a p p} N$, by Lemma 11 , it follows that $C[P[Q / x]] \widehat{\approx}_{\sigma}^{a p p} N$. Hence, by induction hypothesis, $N \Downarrow_{\sigma}$.

Corollary 13. $\left(\widehat{\approx}_{o}^{a p p}\right)_{\Lambda^{0} \times A^{0}}$ is a $\Psi_{o}$-bisimulation.

Proof. We extend $\rightarrow_{0}$ on open terms in such a way that a (possibly open) $\lambda$-term converges if and only if there exists a $\beta$-reduction path to a (possibly open) $\lambda$-term not containing any occurrence of $\Omega$. Then Proposition 12 is applicable.

\section{Final Remarks}

In this paper we introduce the purely syntactical congruence candidate method, but there are also other methods, both syntactical and semantical, for deriving a coinductive characterization of the observational equivalence. We mention the following:

1. Plain induction on computation steps of $\rightarrow_{\sigma}^{*}$. This, purely syntactical, direct approach, which can be traced back to the work of Berry, easily applies to $\rightarrow_{l}$ (see [1]). With suitable extensions in order to take care of open terms, it applies also to $\sigma=h, n$. 
However, it is rather problematic for call-by-value strategies such as $\rightarrow_{v}, \rightarrow_{p}$, or non deterministic strategies like $\rightarrow_{0}$. For a subtle, but complex, proof by induction on computation for $\rightarrow_{v}$ see $[11,10]$.

2. Method based on a Separability algorithm. This method is based on the existence of an effective procedure (see e.g. [2]) which, given two non $\approx_{\sigma}$-equivalent terms, $M, N$, allows to define an applicative context $C$ [] such that either $C[M] \Downarrow_{\sigma}$ and $C[M] \Downarrow_{\sigma}$, or viceversa. To our knowledge, this method works only for $\approx_{h}, \approx_{n}$.

3. Method based on the Domain Logic corresponding to the intersection types presentation of a suitable computationally adequate $C P O-\lambda$-model. This semantical method, introduced in [9], is the generalization of the technique originally used by Abramsky and Ong in [1] for the special case of $\rightarrow_{l}$. In [9], this method is applied to all the strategies of Section 2.

4. Logical Relations method based on a mixed induction-coinduction principle. This semantical method is introduced in [9]. It is the generalization of the technique originally introduced by Pitts ([12]) for $\rightarrow_{l}$ and $\rightarrow_{v}$. In [9], this method is applied to all the strategies of Section 2. The method in [3] for $\rightarrow_{v}$ can be viewed as a weaker variant.

In general, syntactical techniques are more elementary and conceptually simpler than semantical ones, but they are often "ad hoc". However, the general version of the congruence candidate method in this paper, still maintaining the low conceptual complexity of syntactical methods, is much more uniform than plain induction on computation steps. Moreover, it seems to be at least as powerful as the semantical methods in [9].

\section{References}

1. S.Abramsky, L.Ong, Full Abstraction in the Lazy Lambda Calculus, Information and Computation, 105(2):159-267, 1993.

2. H.Barendregt, The Lambda Calculus, its Syntax and Semantics, North Holland, Amsterdam, 1984.

3. L.Egidi, F.Honsell, S.Ronchi Della Rocca, Operational, denotational and logical Descriptions: a Case Study, Fundamenta Informaticae, 16(2):149-169, 1992.

4. F.Honsell, M.Lenisa, Final Semantics for untyped $\lambda$-calculus, M.Dezani et al. eds, TLCA'95 Springer LNCS, 902:249-265, Edinburgh, 1995.

5. F.Honsell, S.Ronchi Della Rocca, An approximation theorem for topological lambda models ..., J. of Computer and System Sciences, 45(1):49-75, 1992.

6. D.Howe, Equality in Lazy Computation Systems, 4th LICS Conference Proceedings, IEEE Computer Society Press, 198-203, 1989.

7. D.Howe, Proving Congruence of Bisimulation in Functional Programming Languages, Information and Computation, 124(2):103-112, 1996.

8. M.Lenisa, Final Semantics for a Higher Order Concurrent Language, CAAP'96 Conference Proceedings, H.Kirchner ed., Springer LNCS, 1059:102-118, 1996.

9. M.Lenisa, Semantic Techniques for Deriving Coinductive Characterizations of Observational Equivalences for $\lambda$-calculi, to appear in TLCA'97 Proc..

10. I.Mason, S.Smith, C.Talcott, From Operational Semantics to Domain Theory, Information and Computation to appear.

11. P.Pérez, An extensional partial combinatory algebra based on $\lambda$-terms, MFCS'91 Conference Proceedings, Springer LNCS, 520, 1996.

12. A.M.Pitts, Relational Properties of Domains, Inf. and Comp., 127:66-90, 1996. 